\title{
LA SARDEGNA MEDIOEVALE NELLA CRONACA DI GIOVANNI DELLA GROSSA \\ (SEC. XV)
}

\author{
MARIA GIUSEPPINA MELONI \\ Istituto sui Rapporti Italo-Iberici \\ (CNR, Cagliari, Italia)
}

La cronaca di Giovanni della Grossa, scritta nella prima metà del Quattrocento, è la più antica tra le cronache della Corsica medioevale' Come si apprende dai dati biografici contenuti all'interno dell'opera stessa, l'Autore, nato nel villaggio di La Grossa, presso Sartene (Corsica sudoccidentale) nel 1388, compì i suoi studi prima a Bonifacio poi a Napoli e conseguì il titolo di notaio; con funzioni di scrivano e di cancelliere fu al servizio dei più importanti personaggi dell'epoca: dapprima del conte di Corsica Vincentello d'Istria, campione della causa aragonese nell'isola (dal 1409 al 1414), poi del governatore genovese Abramo Campofregoso, fino al 1418, quindi di nuovo di Vincentello d'Istria nel periodo in cui quest'ultimo estese la sua autorità su quasi tutto il territorio corso (14191426) e, infine, in seguito a una contoversia che lo oppose al conte di

\footnotetext{
'La cronaca di Giovanni della Grossa, scritta in volgare italiano, partendo dalle origini dei primi abitanti della Corsica si arresta al 1464: venne proseguita fino al 1525 da Pier Antonio Monteggiani, Croniche di Giovanni della Grossa e Pier Antonio Monteggiani, pubbliées par M. l'Abbé Letteron, Bastia, 1919 (d'ora in poi Croniche). A questa edizione faremo riferimento in questo lavoro. Alla seconda metà del XV secolo risale la cronaca, scritta in latino, di Pietro Cirneo, De rebus corsicis, Rerum Italicarum Scriptores, XXIV, Mediolani, 1738, che giunge fino ai primissimi anni del secolo successivo. La storiografia corsa, e in particolare le citate cronache, come ha sottolineato Paul AIMĖs, L'historiographie corse et ses problèmes, in "Melanges de travaux offerts à Clovis Brunel". I. Paris, 1955, presenta diversi e complessi problemi: a tutt oggi gli studiosi di storia della Corsica lamentano la mancanza di un edizione critica di queste opere.

"Anuario de Istudios Medievales". 29 (1999)
} 
Corsica, passò ancora una volta al servizio dei Genovesi. Fu un convinto assertore della legittima appartenenza della Corsica alla Santa Sede, come traspare anche dalle pagine dell'opera, e divenne un sostenitore ed attivo membro del partito pontificio quando l'isola, dal 1444 al 1448, venne governata da commissari papali. In età avanzata si ritirò nel suo villaggio natale, dove redasse o completò la sua cronaca fino alla morte avvenuta, verosimilmente, nel $1464^{2}$.

Nelle vesti di pubblico funzionario egli fu direttamente coinvolto nei principali avvenimenti politici e militari del suo tempo ed ebbe la possibilità di conoscere, leggere e utilizzare documenti di varia natura oggi andati perduti. Il suo interesse per le vicende della storia corsa lo portò, inoltre, come egli stesso sottolinea, a cercare per tutta l'isola "libri antichi" e a raccogliere leggende, tradizioni orali, memorie di "persone vecchie" che utilizzò ampiamente nel racconto degli avvenimenti più remoti ${ }^{3}$.

La cronaca del della Grossa ripercorre la storia della Corsica a partire dalle mitiche e fantasiose origini del suo nome e dei suoi primi abitanti, soffermandosi a lungo sul periodo in cui l'isola fu oggetto di ripetute incursioni e, secondo il cronista, di lunghe occupazioni, da parte degli Arabi. Segue poi nel dettaglio, dall'XI al XV secolo, le intricate vicende della nobiltà insulare, perennemente agitata da odi e contrasti mortali che sconvolsero la Corsica per secoli con la sola parentesi, grazie all'intervento della Santa Sede, che rivendicava i suoi diritti spirituali e temporali sull'isola, di ottant'anni di "buono e pacifico" governo dei Pisani.

${ }^{2} \mathrm{La}$ più recente e completa biografia di Giovanni della Grossa si deve a Jean-André CANCEllieri, voce Della Grossa, Giovamni in Dizionario Biografico degli Italiani, 37, Roma, 1989, pp.68-70. Per il contesto storico nel quale il cronista visse, Pierre ANTONETTI, Histoire de la Corse, Paris, 1973; Histoire de la Corse, sous la direction de Paul Arrighi et Antoine Olivesi, Toulouse, 1971 (nuova edizione 1990). Studi sul notariato in Corsica sono stati condotti da Silio P.P. SCALFATI, Le notariat corse au Moyen Âge d'après les chartriers monastiques, in "Corsica Monastica. Studi di Storia e di Diplomatica", Pisa, 1992, pp.221-254; L'evoluzione del notariato nella Corsica medioevale (secoli XI-XIV), Ibidem, pp.255-266. Lo studioso ipotizza che la preparazione professionale dei notai corsi avvenisse, nella maggior parte dei casi, a Genova e a Pisa. La scelta di Giovanni della Grossa di condurre i suoi studi a Napoli, i cui motivi ci sono ignoti, apparirebbe, dunque, piuttosto inusuale

${ }^{3}$ In un passo della Cronaca il della Grossa si definisce "quello che raccolse libri degni di fede antiqui, per tutta Corsica li ricercava, che tractavano quello che nel presente sta scripto di le cose socciesse in Corsica", Croniche. p. 231. In alcuni punti, inoltre, fa riferimento alle sue fonti, dichiarando di basarsi su "ciò che si dice o si trova scritto", pp. 78-79, 82, 116. Lo stesso cronista afferma che la parte originale della sua opera, basata quindi su fonti dirette e ricordi personali. incomincia con il racconto dei fatti riguardanti il conte Arrigo della Rocca (seconda metà del '300), Croniche, p. 231. 
Sullo sfondo, le lotte tra le potenze che si contendevano il dominio sull'isola e alle quali le diverse fazioni della feudalità corsa di volta in volta si appoggiavano: dapprima le repubbliche marinare di Pisa e di Genova poi, dopo il prevalere di quest'ultima sulla rivale tirrenica, tra la repubblica ligure e la Corona d'Aragona.

Se la parte della cronaca che va dalle origini al XIII secolo è, per giudizio unanime degli storici, caratterizzata da evidenti confusioni cronologiche e da una sovrabbondanza di racconti ispirati a leggende e tradizioni popolari, che prevalgono sui pur presenti elementi di realtà storica $^{4}$, l'opera è senza dubbio una fonte preziosa per la storia della Corsica dei secoli XIV e $\mathrm{XV}^{5}$.

Nelle pagine della sua opera il della Grossa fa riferimento più volte alla vicina Sardegna: emergono, così, attraverso il racconto delle vicende

${ }^{4}$ Pierre Antonetti, Histoire de la Corse, cit., pp. 112-113; Huguette TAviani- Carozzi, Le debuts de la colonisation, in Histoire de la Corse, sous la direction de Paul Arrighi, cit., p.151. Già la storiografia positivista, a partire dalla fỉne dell'Ottocento, sottolineò I'inattendibilità della Cronaca, soprattutto riguardo ai periodi più antichi fino a tutto l'alto Medioevo, giudicandola piena di leggende e di evidenti confusioni cronologiche (LETTERON, Introduction a Croniche, cit., pp. IX-XIX; IDEM, Introduction a Histoire de la Corse comprenant la description de cette ile d'après A. Giustiniani, les chroniques de Giov. della Grossa et de Monteggiani remaniées par Ceccaldi, la chronique de Ceccaldi et la chronique de Filippini, traduction française de M. I'Abbé Letteron, Bastia, 1888, pp. XIX-XL; H. YVIA CROCE, Giovanni della Grossa in "Anthologie des écrivains corses", I, Ajaccio, 1929, pp.3638. A partire dagli anni 80 di questo secolo l'opera del della Grossa è stata oggetto di un processo di rivalutazione tendente a metterne in evidenza la ricchezza soprattutto come fonte etnografica. In particolare, una corrente di storici-sociologi ha cercato di attibuire alla Cronaca un valore di fonte per la storia sociale della Corsica anche per l'alto Medioevo. Antoine CASANOVA, Révolution féodale, pensée paysanne et caractères originaux de l'histoire sociale de la Corse. "Études corses", 15 (1980). pp.19-78. L'approccio etno-sociologico alla Cronaca è stato però criticato da Silio P.P. SCALFATI, Stranieri nella Corsica medioevale, in "Dentro la città. Stranieri e realtà urbane nell'Europa dei secoli XII-XVI", a cura di Gabriella Rossetti, Napoli, 1989, pp.111-119, che, sottolineando il rischio di ricavare, attraverso questo tipo di approccio, un'immagine distorta e parziale del Medioevo corso, afferma decisamente la necessità di una più attiva e rigorosa ricerca e studio delle fonti archivistiche relative alla Corsica medioevale e moderna, dovunque siano prodotte.

${ }^{5}$ Com'è noto, la storia della Corsica medioevale soffre di una grave carenza di fonti. Alla penuria di documentazione indigena si supplisce, almeno in parte, con le notizie provenienti da fonti esterne $o$, in tempi più recenti, con $i$ risultati degli scavi archeologici. Per una ricostruzione del Mediovo corso sulla base delle fonti esistenti si vedano i capitoli dedicati a questo periodo (Huguette Taviani-Carozzi, La Corse, terre de Saint Pierre e Les debuts de la colonisation; René Emmanuell, L'implantation génoise) in Histoire de la Corse, cit., pp. 129-182. Di grande interesse per la storia non solo ecclesiastica, ma anche sociale della Corsica nei secoli XI e XII sono gli studi condotti da Silio P.P. SCALFATI sulla documentazione dell'archivio della Certosa di Calci (Pisa), riguardante i monasteri benedettini corsi affiliati al monastero di San Gorgonio dell'isola della Gorgona e raccolti nei volumi Corsica monastica, cit.; Diplomatica corsa, Pisa, 1994; La Corse médiévale, Ajaccio, 1996. 
della storia corsa, episodi e momenti di storia sarda. Le due isole ebbero, nei secoli altomedioevali, sorte comune: entrambe subirono l'invasione vandala, la dipendenza dall'impero bizantino, il tormento delle incursioni saracene. A partire dal IX secolo la loro storia si differenzia nettamente: mentre la Sardegna, isolata nel Mediterraneo a causa della presenza araba, maturerà l'originale esperienza dei giudicati e, dopo la parziale fine di questa, la sua storia verrà caratterizzata dalla presenza di altre entità politiche forti (oltre al superstite giudicato d'Arborea, il Comune di Pisa e poi la Corona d'Aragona), la Corsica, più vicina al continente italiano, manterrà con questo più stretti contatti, ma la sua storia politica, nella quale si inserisce, a partire dalla fine del XII secolo, la presenza coloniale genovese, verrà caratterizzata da un cronico vuoto di potere - solo momentaneamente colmato dai periodici interventi della Santa Sede- che determinerà il succedersi di lotte tra $\mathrm{i}$ deboli potentati locali, non adeguatamente supportati dalle potenze che avevano interessi strategici sull'isola. Neppure la comune infeudazione pontificia al re d'Aragona Giacomo II il Giusto (1297), porterà all' unificazione delle sorti delle due isole, dal momento che il dominio aragonese sulla Corsica rimarrà solo nominale

Nonostante la vicinanza geografica, le due isole non ebbero, nel Medioevo, intensi rapporti che furono limitati, quasi esclusivamente, agli scambi commerciali e umani che avvenivano tra il sud della Corsica ed il nord della Sardegna. Nelle pagine della cronaca di Giovanni della Grossa quest'ultima ha, tutto sommato, un ruolo abbastanza marginale; cercheremo, tuttavia, di esaminare i motivi ed i modi della presenza della Sardegna nella narrazione del cronista corso.

La prima menzione della Sardegna per quanto riguarda il periodo medioevale $^{7}$ si inserisce nel racconto degli attacchi arabi alle coste del Mediterraneo centrale e concerne l'origine dei quattro giudicati e della rivalità tra le repubbliche di Pisa e di Genova per il predominio nell'isola. Il cronista, che colloca gli avvenimenti al tempo del papa Gregorio IV (827-

"Per un inquadramento generale della storia medioevale della Sardegna, Francesco Cesare Casula, La storia di Sardegna, Sassari-Pisa, 1992, pp. 127 e ss.; per la Corsica, Histoire de
la Corse. cit. Un raffronto tra le vicende storiche delle due isole è nel saggio di Arrigo Solmi. La Corsica. Studio storico, "Archivio Storico di Corsica". I (1925), pp. 4-38.

${ }^{7}$ In realtà Giovanni della Grossa cita per la prima volta la Sardegna accomunandola alla Corsica nei modi e nei tempi del loro primo popolamento: le due isole, racconta il cronista. vennero popolate pressocchè contemporaneamente da due mitici personaggi chiamati Cor e Sardo all epoca della fuga di Enea da Troia, Croniche, p.4. 
844), narra che, subito dopo aver assediato Roma ed essere stati respinti dalle forze terrestri e marittime dei Pisani, dei Genovesi e del conte di Barcellona, carichi di bottino i Mori si erano diretti verso l'Africa. Per ordine del Papa dodici galere pisane e quattro genovesi che avevano partecipato alla difesa di Roma avevano inseguito i Musulmani fino in Sardegna, dove questi erano da tempo insediati nelle città di Cagliari e di Alghero ${ }^{8}$ : le navi pisane e genovesi avevano dunque di sorpresa attaccato le due città sarde, liberandole dall'occupazione saracena. Da quel momento, Pisani e Genovesi cominciarono a spartirsi l'isola "e se ne fecero quattro parte, le tre a Pisani, e una a Genovesi, e si chiamava quattro Giudicati, cioé il Giudicato d'Arborea, e il Giudicato di Callari, e il Giudicato di Gallura; e a ciascheduno di questi si messe un Judice pisano. Il quarto judicato fu Locodoro, dove posero un giudice genovese, secondo la quantità delle galere". Il della Grossa continua poi affermando che, al di sopra di ogni giudice, stava un governatore inviato dal papa, al quale ultimo spettava l'autorità suprema sull'isola, feudo della Chiesa; aggiunge, però, che ben presto i Papi cessarono di inviare il loro rappresentante e i giudici governarono da soli, fino a quando le due repubbliche marinare cominciarono a combattersi per ottenere il predominio nell' isola ${ }^{9}$.

E' evidente, innanzittutto, in questo racconto, una certa confusione cronologica, del resto, come si è detto, molto frequente nella cronaca per tutto l'alto Medioevo. Se da una parte l'origine dei giudicati sardi sembra effettivamente risalire proprio al IX secolo, quando, in concomitanza con l'occupazione araba della Sicilia (827), che provocò il distacco definitivo della Sardegna da Bisanzio, i quattro lociservatores delle mereie o parti di Calari, Torres, Gallura e Arborea cominciarono a governare come sovrani indipendenti ${ }^{10}$, d'altra parte è noto che l'impresa contro gli Arabi in seguito alla quale Pisani e Genovesi iniziarono la loro penetrazione politica ed

\footnotetext{
${ }^{8}$ In realtà non esistono attestazioni documentarie sull'esistenza di questa città prima dell XII secolo, Rosalind BRown, Alghero prima dei Catalani, in "Alghero, la Catalogna e il Mediterraneo. Storia di una città e di una minoranza catalana in Italia (XIV-XX secolo)", a cura di Antonello Mattone e Piero Sanna, Sassari, 1994, pp. 49-58. Per quanto riguarda Cagliari, invece, l'antica Caralis romana, ubicata sulla riva del mare, venne abbandonata tra l'VIII e il $\mathrm{X}$ secolo proprio in seguito alle continue scorrerie arabe, e gli abitanti si trasferirono in un sito più protetto tra gli stagni, dove sorse la città di Santa Igia, che divenne la capitale del giudicato di Calari, Francesco Cesare Casula, La storia di Sardegna, cit., pp. 160, 190.

${ }^{9}$ Croniche, pp. 56-57.

${ }^{10}$ Francesco Cesare Casula, La storia di Sardegna, cit., pp. 136-183.
} 
economica in Sardegna fu quella compiuta nel 1015-1016 contro Mugiâhid signore di Denia e delle Baleari, ed è a quest'impresa, presumibilmente, che il nostro cronista fa riferimento, collocondola però circa due secoli prima" ${ }^{11}$.

In secondo luogo, è interessante notare come il della Grossa si discosti, per una variante di non poco conto, dalla tradizione storiografica pisana secondo la quale, in base ad un accordo tra le due repubbliche marinare, in seguito alla vittoria su Mugiâhid il possesso della Sardegna sarebbe andato ai Pisani, che vi istituirono, nel corso dell'XI secolo, i quattro giudicati, mentre i Genovesi si sarebbero accontentati del bottino di guerra sottratto agli Arabi ${ }^{12}$. D'altra parte, la versione di Giovanni della Grossa che, come si è visto, attribuisce alle due repubbliche marinare una spartizione dell'isola in base alle forze marittime messe in campo contro gli Arabi, e l'insediamento dei Genovesi in un giudicato, quello di Torres o Logudoro, non si ritrova nemmeno nelle principali cronache genovesi che, nel medesimo tentativo di giustificare le pretese della repubblica ligure sull'isola, esaltavano la parte avuta da Genova nella sconfitta del re moro, che sarebbe stato portato prigioniero in questa città, e scrivevano che $\mathrm{i}$ Genovesi avevano sottomesso la Sardegna e si erano insediati al governo del giudicato di Cagliari, "tunc caput totius Sardiniae"13.

\footnotetext{
"Per quanto riguarda gli altri elementi del racconto, le biografie del papa Gregorio IV parlano di attacchi saraceni alle coste del Lazio, che indussero il pontefice ad ordinare la costruzione di un borgo fortificato presso il porto di Ostia, mentre un sacco di Roma avvenne nell'846, sotto il pontificato del successore Sergio II, Dizionario Storico del Papato, diretto da Philippe Levillain, 2 voll., Milano, 1996; il conte di Barcellona, poi, che secondo il cronista sarebbe stato protagonista anche della lotta contro i Mori di Corsica, ricorda la figura di Raimondo Berengario III, vissuto però tre secoli dopo i fatti narrati, che fu promotore di numerose imprese contro i Saraceni, in particolare di quella compiuta, insieme con i Pisani, contro i Mori delle Baleari nel 1113 e celebrata nel Liber Maiorichinus. Sulle incursioni arabe in Sardegna Alberto Boscolo, Studi sulla Sardegna bizantina e giudicale, Cagliari, 1985.

${ }^{12}$ Questa versione, con qualche piccola variante, si trova nelle cronache del XII-XIV secolo, come quelle di Bernardo Maragone, Annales Pisani, a cura di M. Lupo Gentile, Rerum Italicarum Scriptores, tomo VI, parte II; nella Cronaca di Pisa di Ranieri Sardo, a cura di Ottavio Banti, Roma, 1963, e nella cronaca pisana del XIV secolo, conservata nell'Archivio di Stato di Lucca, esaminata da Ottavio BANTI, Studio sulla genesi dei testi cronistici pisani del XIV secolo, "Bullettino dell'Istituto Storico Italiano per il Medio Evo e Archivio Muratoriano", 75 (1963), pp. 259-319. I primi ad attribuire ai Pisani l'istituzione dei quattro giudicati furono, come afferma Enrico BESTA, La Sardegna medioevale (2 voll., Palermo, 1908-1909), 1, 1908, pp. 56-67; II, 1909, pp. 7-14, Jacopo della Lana ed altri commentatori di Dante; una raccolta dei loro scritti sull'argomento in Filippo VIVANET, La Sardegna nella Divina Commedia e nei suoi commentatori, Sassari, 1879.

${ }^{13}$ Annali Genovesi di Caffaro e de' suoi continuatori, a cura di Luigi Tommaso BelgranoCesare Imperiale di Sant'Angelo (5 voll., Roma, 1890-1929), I, pp. 161, 197.
} 
E' possibile che il della Grossa abbia conosciuto le cronache pisane; non sappiamo, però, quali siano state le sue fonti per la versione da lui riportata, che potrebbe anche essere stata tratta da un testo andato perduto. Non bisogna dimenticare, inoltre, che il nostro cronista fu a lungo al servizio dei Genovesi durante la sua attività di notaio e cancelliere, e che ebbe la possibilità di consultare testi e documenti, anche questi, presumibilmente, oggi scomparsi, che potrebbero aver influito sulla sua versione riguardo al ruolo avuto da Genova in Sardegna ed ai suoi diritti sul giudicato di Torres. E' certo comunque che, ancora ai tempi del della Grossa, si aveva memoria degli antichi rapporti politici ed economici che avevano legato questo giudicato e la città di Sassari alla repubblica ligure, di cui un segno tangibile era la presenza della famiglia genovese dei Doria, che intorno al XII secolo avevano fondato le città di Alghero e Castelgenovese (da essi posseduta ancora nella prima metà del XV secolo) e che ebbero una parte da protagonisti sia nella storia del giudicato turritano, che in epoca catalanoaragonese $\mathrm{e}^{14}$.

Al di là degli interrogativi -ai quali è difficile dare una rispostasulle fonti della versione di Giovanni della Grossa sull'origine dei giudicati sardi, è singolare il fatto che egli, diversamente dagli altri cronisti che ne hanno parlato, collochi questo avvenimento nel IX secolo, ossia nell'epoca in cui esso effettivamente si verificò; il suo racconto, inoltre, è interessante perchè da esso emerge la conoscenza che si aveva, nella Corsica del Quattrocento, di un importante periodo della storia sarda quale quello giudicale.

Giovanni della Grossa conclude questa parentesi riguardante le vicende della Sardegna con un' interpretazione dell'origine della lingua sarda, che sembra rimarcare una certa difficoltà di rapporti tra le due isole: egli afferma che molti di coloro che avevano partecipato alla spedizione contro i Mori, appartenenti a varie nazionalità: navarresi, guasconi, francesi,

\footnotetext{
${ }^{14}$ Sui rapporti tra il giudicato di Torres e Genova, Francesco Cesare CASULA, La storia di Sardegna, cit., pp. 217-253; Geo PISTARINO, Genova e la Sardegna nel XII secolo in "La Sardegna nel mondo mediterraneo", I Convegno internazionale di studi geografico-storici, 2, a cura di Manlio Brigaglia, Sassari, 1981, pp.33-125; Documenti inediti sui traffici commerciali tra la Liguria e la Sardegna nel secolo XIII a cura di Nilo Calvini, Evandro Putzulu, Vanna Zucchi, Padova, 1957; su Sassari e i suoi rapporti con la repubblica ligure Angelo Castellaccio, Sassari medioevale, I, Sassari, 1996; sul ramo "sardo" dei Doria Genealogie medioevali di Sardegna (a cura di Leonard L. Brook, Francesco Cesare Casula, Maria Mercè Costa, Annamaria Oliva, Romeo Pavoni, Marco Tangheroni), Cagliari-Sassari, 1984, tav.XIXXX-XXI.
} 
italiani, nonchè molti mori che si erano convertiti al cristianesimo, si fermarono nell'isola, e vi stabilirono il proprio domicilio. Dal miscuglio dei linguaggi parlati da tutte queste genti ebbe origine "una sorta di parlare brutto in Sardigna che non si accorda con nesciuna nazione", una lingua dunque diversa da qualunque altra e incomprensibile agli stranieri ${ }^{15}$.

Nelle pagine della cronaca la Sardegna compare spesso come luogo di esilio o come rifugio di persone perseguitate che cercavano riparo dalle avverse fortune politiche ${ }^{16}$. Il primo caso in cui l'isola svolge questo ruolo riguarda l'esilio in Gallura dei signori di Cinarca, una delle principali famiglie feudali dell'Oltremonti (sud-ovest della Corsica) ${ }^{17}$, che sarebbe durato, secondo la cronologia del della Grossa, dal 1072 al 1112, e che offre ancora un interessante scorcio della Sardegna giudicale.

Narra Giovanni della Grossa che il conte Andrea di Cinarca ed i suoi familiari, esiliati in Sardegna dal marchese di Massa, che era stato inviato in Corsica dal papa Gregorio VI in qualità di governatore per porre un freno all'anarchia che vi regnava ${ }^{18}$, si trovavano in grande povertà. L'unico figlio dodicenne del conte Andrea, di nome Arrigo, si mise allora al servizio, come stalliere, del giudice di Gallura "il quale era gentilhomo pisano". Le sue doti consentirono al giovane corso di farsi strada alla corte giudicale: dalla stalla passò a servire alla mensa del giudice, poi grazie alla sua abilità

${ }^{15}$ Croniche, p. 57. Anche Arrigo Solmi nel suo saggio La Corsica, cit, pp. 12-13, sottolinea come la diversità del percorso storico seguito dalle due isole si esprima in modo evidente nel linguaggio: mentre il sardo è considerato dai linguisti una vera e propria lingua, il corso non è che una varietà tra $\mathrm{i}$ dialetti italiani.

${ }^{16}$ La Sardegna fu terra di esilio per lo stesso Giovanni della Grossa che, intorno al 1414 fu costretto da alcuni signori ribelli a Vincentello d'Istria, a rifugiarsi nell'isola, dove venne accolto alla corte del marchese di Oristano Leonardo Cubello, Croniche, pp. 259-260.

${ }^{17}$ Con il termine di Oltremonti o Pưmonte (o Banda di fuori) veniva definita dai Genovesi "secondo un'attibuzione terminologica essa stessa di essenza coloniale o per lo meno metropolitana" la parte sud-occidentale della Corsica, separata da una dorsale montuosa che divide l'isola secondo una direzione nord-ovest sud-est, dalla parte nord-orientale detta Cismonti (o Banda di dentro), Jean André CANCELliERI, Corsi e Genovesi: elementi per una fenomenologia della colonizzazione nel Mediterraneo medioevale, in "Sardegna, Mediterraneo e Atlantico tra Medioevo ed Età Moderna. Studi in onore di Álberto Boscolo", II, Milano, 1992, pp.405-423. In questo stesso lavoro notizie sulle principali signorie dell'Oltremonti (quelle dei Leca, dei Della Rocca, d'Istria, dette dei Cinarchesi. Come sottolinea l'Autore, non esiste a tutt'oggi un storia dinastica delle casate cinarchesi nel basso Medioevo, Ibidem, pp.415-416).

${ }^{18}$ Sulla presenza in Corsica dei marchesi di Massa, Silio P.P. SCALFATI, Un placito nella storia della Corsica medioevale in "Corsica monastica", cit., pp. 21-42. La prima attestazione documentaria della presenza nell'isola di un marchese di Massa (il marchese Alberto Rufo), come dimostra Scalfati, risale agli anni 1070-1080 e coincide, più o meno, con la datazione data dal della Grossa. 
a cavallo, divenne uomo d'armi. La benevolenza e la stima del sovrano gallurese giunsero al punto che questi gli volle dare in moglie una sua sorella vedova, di nome Cicha, che era venuta a trovarlo da Pisa e quando, dopo la morte del marchese di Massa e l'insediamento dei Pisani in Corsica, si presentò ad Arrigo l'occasione per riappropriarsi delle sue terre, che nel frattempo erano state occupate da altri signori, il giudice gli diede un'imbarcazione e degli uomini che lo aiutassero nell'impresa. Dopo il ritorno in Corsica, il figlio di Arrigo e della nobildonna pisana, nato e cresciuto in Sardegna, fu conosciuto con l'appellativo di "il Sardo" 19 .

Le notizie riguardanti l'esilio in Sardegna dei Cinarchesi sarebbero state tratte, come afferma lo stesso cronista, oltre che dalla tradizione orale anche da documentazione scritta ("e tale che si dicie e trova scripto"): è possibile che egli abbia avuto conoscenza di testi e documenti, di cui non è rimasta traccia, conservati negli archivi privati di questa famiglia che, secondo la tradizione, discendeva da Arrigo Bel Messere, leggendario capostipite della feudalità corsa. La famiglia dei signori di Cinarca, così come le altre famiglie feudali dell'isola, tendevano a costruirsi una tradizione familiare che ricollegasse la loro origine a quella di nobili eroi, al fine di legittimare il loro potere e le loro ambizioni; il racconto del lungo esilio in Sardegna dei Cinarchesi potrebbe aver avuto, in questo contesto, come afferma il Casanova ${ }^{20}$, la funzione di riempire gli ampi vuoti cronologici tra gli anni intorno al Mille e i secoli XII-XIII, periodo in cui maggiore fu la potenza e la fama di questa casata.

E' difficile, se non impossibile, datare l'origine di questa tradizione, e tantomeno siamo in grado di chiarire, attraverso la scarsa documentazione relativa al giudicato di Gallura, se essa abbia o no un fondamento storico. In ogni caso, il racconto è interessante perché rivela il prestigio di cui godeva in Corsica l'istituzione giudicale sarda: il giudice di Gallura è senz'altro un personaggio potente e prestigioso, capace di fornire appoggi politici, sostegno economico e militare alla famiglia corsa in difficoltà, che poteva vantare, nel costruirsi la sua tradizione familiare, i legami e la protezione ottenuta. Dall'episodio traspare l'immagine di una Sardegna prospera e tranquilla, in contrapposizione alla situazione di disordine e anarchia dominanti in Corsica. Il cronista, che ribadisce il ruolo avuto dai

\footnotetext{
${ }^{19}$ Croniche, pp. 99-102; 116-120.

${ }^{20}$ Antoine Casanova, Révolution féodale, cit., pp. 28-29 e nt.66.
} 
Pisani nella nascita e nel governo dei giudicati sardi (il giudice di Gallura è infatti un gentiluomo pisano), traccia inoltre, attraverso piccoli dettagli della quotidianità (la presenza di personale addetto alle stalle, di valletti che servivano alla mensa del giudice ecc.) un quadro della vita che si svolgeva presso la corte giudicale gallurese, che appare come una corte semplice ma aperta e disponibile all'accoglienza degli stranieri e ai rapporti con il continente italiano. Come si è detto, non esiste alcuna prova documentaria che possa convalidare la tradizione riportata dal della Grossa ed i particolari del suo racconto; tuttavia la notizia della presenza nel regno gallurese, in quegli anni, di un giudice pisano, si ritrova pure nella storiografia sarda del Cinquecento ${ }^{21}$.

Nel racconto delle vicende della Corsica nell'XI secolo la Sardegna compare ancora in un avvenimento luttuoso che, secondo il nostro cronista, fu foriero di gravi conseguenze per l'isola: l'assassinio del conte Arrigo Bel Messere, mitico eroe, pacifico e giusto, che sarebbe stato ucciso a tradimento, nell'anno Mille, da un Sardo assoldato dalla famiglia dei signori Talaventacci, a lui ostili ${ }^{22}$. La figura di Arrigo Bel Messere fa parte del patrimonio di leggende popolari, la cui origine è difficile datare, al quale Giovanni della Grossa attinse a piene mani quando redasse la sua Cronaca $^{23}$; la collocazione della sua morte nell'anno Mille è un sintomo di quella mentalità che tendeva a vedere, in concomitanza con il passaggio del millennio, il verificarsi di eventi eccezionali, di manifestazioni della volontà divina che avrebbero segnato il corso della storia. Con la morte di Arrigo Bel Messere, infatti, avrebbe avuto origine il disordine feudale che caratterizzò poi tutto il Medioevo corso ${ }^{24}$.

Il motivo dell'attribuzione ad un Sardo dell'esecuzione di questo delitto va cercato, verosimilmente, nella particolare fama di cui gli abitanti

\footnotetext{
"Giovanni Francesco FARA, il primo storico della Sardegna, nel suo De rebus sardois, pubblicato nel 1580 (ed. a cura di Enzo Cadoni, Sassari, 1992, libro II, pp. 310-311), cita tra i primi giudici di Gallura un Constantinus Gerardescus (Gherardesca?) al quale Gregorio VII avrebbe inviato una lettera nel 1074, e che potrebbe identificarsi nel gentiluomo pisano di cui parla il della Grossa. Questo giudice e gli altri personaggi presenti nel racconto di Giovanni della Grossa (la sorella del giudice Cicha, erroneamente definita figlia, e Arrigo di Cinarca) sono inseriti, a titolo di ipotesi, nelle Genealogie medioevali, cit., tav. IV, lemmi 3 e 4.

"Croniche, pp.69-70.

${ }^{23}$ Secondo Pierre ANTONETTI, Histoire de la Corse, cit., pp. 113-114, la leggenda sarebbe nata nel XIII secolo ispirata dai racconti dell'epopea carolingia.

${ }^{24}$ Sull'interpretazione della leggenda di Arrigo Bel Messere e sul suo ruolo nell'opera del della Grossa Huguette Taviani- CAROzZI, La Corse, terre de Saint Pierre, cit., pp. 146-148.
} 
dell'isola godevano, nel Medioevo, riguardo all'abilità nell'uso di un'arma micidiale ed infallibile, la "verga sardesca", con la quale, appunto, secondo il racconto della cronaca, venne ucciso Arrigo Bel Messere. L'episodio, nonostante la sua mancanza di fondamento storico, rivela, dunque, come in Corsica fosse da lungo tempo conosciuta ed usata un'arma tipicamente sarda, nell'uso della quale i Sardi erano considerati maestri ${ }^{25}$.

La centralità della Sardegna nelle lotte che nel XII secolo scoppiarono tra le città marinare di Pisa e di Genova è chiaramente individuata da Giovanni della Grossa nella parte della cronaca dedicata all'epoca della cosidetta "pax pisana", che costituisce per il cronista il periodo più felice della storia della Corsica ${ }^{26}$. Secondo il della Grossa il buon governo dei Pisani ebbe inizio al tempo del papa Urbano (presumibilmente Urbano II, 1088-1099) e durò cento anni: ottant'anni di prosperità in cui "non socciesse cosa nuova da notare se non buona pace e grande benefitio universale" per tutta l'isola, seguiti da vent'anni durante i quali l'acuirsi della rivalità tra Pisani e Genovesi per il predominio sulla Sardegna ebbe i suoi riflessi negativi anche sulla Corsica. Le due città si contesero infatti il possesso di Bonifacio, che secondo il della Grossa rivestiva per entrambe una particolare importanza oltre che per essere un ottimo porto, anche perché situato a breve distanza sia dal giudicato di Gallura, governato dai Pisani, che da quello di Logudoro, "che era di ragione di Gienovesi"27. L'insediamento dei Genovesi a Bonifacio, che il

\footnotetext{
${ }^{25}$ Sulle caratteristiche e sulla diffusione di quest'arma nel bacino del Mediterraneo vedi l'ampio e documentato studio di G. FoIS, Un'arma medioevale sarda: la "virga", "Quaderni Bolotanesi", 21 (1995), pp. 183-220.

${ }^{26} \mathrm{~L}$ 'influenza religiosa, politica ed economica di Pisa in Corsica, iniziata con la penetrazione di ordini monastici toscani, si consolidò quando, nel 1077, il papa Gregorio VII concesse il vicariato apostolico nell'isola al vescovo pisano Landolfo, e durò per circa due secoli, senza cessare del tutto nemmeno dopo la sconfitta di Pisa da parte di Genova nella battaglia della Meloria nel 1284. Su questo periodo della storia corsa Cinzio ViolanTE, Le concessioni pontificie alla Chiesa di Pisa riguardanti la Corsica alla fine del secolo XI, "Bullettino dell'Istituto Storico Italiano per il Medioevo e Archivio Muratoriano", 75 (1963); Silio P. P. SCALFATI, I Benedettini e la "pax pisana" in Corsica, in "Corsica monastica", cit., pp.151-158; Jean André CANCELlIERI, De la "Corse pisane" a la "Corse génoise": remarques sur la portée structurelle insulaire de la bataille de la Meloria (1284), in "Genova, Pisa e il Mediterraneo tra Due e Trecento. Per il VII centenario della battaglia della Meloria. Atti della Società Ligure di Storia Patria", n.s., vol. XXIV (1984), fasc. II, pp. 571-83.

${ }^{27}$ Croniche, pp. 114-122. Il racconto del della Grossa, mettendo la Sardegna al centro dei conflitti tra Pisa e Genova, sminuisce in qualche modo l'importanza che anche la Corsica ebbe in questi conflitti, causati dalle concessioni pontificie alla Chiesa di Pisa riguardo a quest'ultima tra il 1077 e il 1091; conflitti che portarono il papa Innocenzo II, nel 1133, alla divisione dei vescovadi e dell'isola stessa in due zone d'influenza, Cinzio Violante, Le concessioni
} 
cronista colloca giustamente nel $1195^{28}$, segnò l'inizio del declino di Pisa e del predominio della repubblica ligure sulla Corsica.

Un largo spazio occupa nella cronaca il racconto delle lotte feudali che videro protagonista, nella seconda metà del XIII secolo, un esponente della famiglia dei Cinarchesi, Sinucello della Rocca detto Giudice di Cinarca il quale, tentando di mantenersi in un precario equilibrio tra Pisa e Genova, si impose sugli altri signori corsi costituendosi un vasto dominio personale ${ }^{29}$.

Nel racconto di questi avvenimenti la Sardegna è ricordata come luogo di esilio per gli oppositori del signore corso e come una delle mete di un forte flusso migratorio che, in quel periodo tormentato, provocò lo spopolamento di vaste zone della Corsica ${ }^{30}$. Come si rileva anche dall'opera del della Grossa, la Gallura, separata da quest'isola dal breve tratto di mare

pontificie, cit.; Giuseppe SCALIA, La consacrazione della cattedrale sullo sfondo del contrasto con Genova per $i$ diritti metropolitani sulla Corsica, in "Nel IX centenario della metropoli ecclesiastica di Pisa", Atti del Convegno di Studi (Pisa, 1992), Pisa, 1995, pp.131-141; Silio P.P. SCALFATI, Le diocesi suffraganee corse, Ibidem, pp. 235-247.

${ }^{28}$ Croniche, p. 121. Sulla nascita e lo sviluppo della colonia ligure di Bonifacio Jaques HEERS, Un exemple de colonisation médiévale: Bonifacio au XIII siécle "Anuario de Estudios Medievales", 1 (1964), pp.561-571; Roberto Sabatino LOPEZ, Da mercanti ad agricoltori. aspetti della colonizzazione genovese in Corsica, in "Homenaje a Jaime Vicens Vives", I, Barcelona, 1965, pp. 535-532; Giovanna PETTI BALBI, Genova e Corsica nel Trecento, Roma, 1973; Jean André CanCELLIERI, Bonifacio au Moyen Âge, Ajaccio, 1997.

${ }^{29}$ Su questo personaggio Jean André CANCELLIERI, voce Della Rocca, Sinucello (detto Giudice di Cinarca) in Dizionario Biografico, cit., pp. 322-326. Il nome-titolo di "giudice", secondo Cancellieri, potrebbe derivare dall'appellativo che, in Corsica, dall'Alto Medioevo, designava "i rappresentanti per eccellenza del potere pubblico o i detentori di un potere di tipo eminente", ma potrebbe anche essere "una contaminazione o un'imitazione diretta dei titoli giudicali della Sardegna”, Ibidem, pp. 322-23.

${ }^{30}$ Croniche, p. 155 . Sulla situazione demografica della Corsica nei secoli XIII-XIV, Franco Borland, La popolazione della Corsica nel Medioevo, "Archivio Storico di Corsica", XVI (1940), n.1, pp.13-42; Jean André CANCELLIERI, Directions de recherche sur la démographie de la Corse médiévale (XIII-XV siècles) in "Strutture familiari, epidemie, migrazioni nell'Italia medioevale”, a cura di Rinaldo Comba, Gabriella Piccini, Giuliano Pinto, Napoli, 1984, pp. 401-433. Sul fenomeno dell'emigrazione corsa nelle varie parti d'Italia, determinata oltre che dall'endemico stato di anarchia anche dalla scarsità delle risorse economiche. Ildebrando IMBERCIADORI, Corsi in Maremma nella seconda metà del Quattrocento, "Archivio Storico di Corsica”, VII (1931), n.2, pp. 204-224; Geo PISTARINO, Una colonia corsa a Campiglia Marittima nel Quattrocento, "Bollettino Storico Livornese", IV (1940), n. 4; Giovanna PETTI BALBI, I corsi a Genova (III capitolo del volume Genova e Corsica, cit., pp. 135-166); Huguette TAVIaNi-CAROzZI, Les debuts, cit., pp.177 e ss. per la presenza corsa a Pisa; Anna EsPosITo, Una minoranza e il suo insediamento: i Corsi, in "Un'altra Roma. Minoranze nazionali e comunità ebraiche tra Medioevo e Rinascimento", Roma, 1995, pp. 93-106; Jean André CANCELLIERI, Emigrer pour servir: la domesticité ds femmes corses en Italie comme rapport de dépendance insulaire (1250-1350 environ). in "Coloniser au Moyen Âge". a cura di Michel Balard e Alain Ducellier, Paris, 1995. 
delle Bocche di Bonifacio, e il Logudoro, furono in ogni tempo le zone maggiormente interessate a movimenti migratori, temporanei o stabili, di Corsi alla ricerca di migliori condizioni di vita, lontano dalle guerriglie, dalle carestie e dalla miseria della loro terra. E' possibile che, nella seconda metà del XIII secolo, una forte attrazione venisse esercitata soprattutto dalla città di Sassari che, in quegli anni, dopo essersi costituita in Comune dapprima sotto l'egida pisana, poi genovese, godette di un notevole sviluppo economico ${ }^{31}$.

Con la morte di Giudice di Cinarca, catturato e imprigionato dai Genovesi nel 1312, la narrazione di Giovanni della Grossa giunge al XIV secolo.

La storia corsa del Tre e Quattrocento è caratterizzata dai ripetuti tentativi di Genova di imporre a tutta l'isola il proprio dominio, saldamente assodato solo nelle due colonie di Calvi e Bonifacio, e dal coinvolgimento della Corsica nelle lotte tra Genova e la Corona d'Aragona, che si acuirono dopo la creazione, ad opera del pontefice Bonifacio VIII, del teorico regno formato dalle due isole, infeudato nel 1297 al re aragonese Giacomo II. Questo avvenimento, e l'inizio della conquista della Sardegna pisana da parte dei Catalano-Aragonesi nel 1323, determinarono la formazione in Corsica di una fazione di nobili filoaragonesi, facente capo alla famiglia dei signori di Cinarca, ed il conseguente aggravamento del disordine e dell'anarchia che regnavano nell'isola ${ }^{32}$.

\footnotetext{
${ }^{31}$ Marco TANGHERONI, Nascita ed affermazione di una città: Sassari dal XII al XIV secolo in "Gli Statuti sassaresi. Economia, società, istituzioni a Sassari nel Medioevo e nell'Età Moderna", a cura di Antonello Mattone e Marco Tangheroni, Cagliari, 1986, pp. 45-63; Angelo CASTEllaCCIO, Sassari medioevale, cit. Sulla presenza corsa nella città, Maria Giuseppina Meloni, Presenza corsa a Sassari a metà del 1300, "Medioevo. Saggi e Rassegne” 13 (1988), pp. 9-33.

"Sull'infeudazione del regno di Sardegna e Corsica a Giacomo II d'Aragona, Vicente SALAVERT y ROCA, Cerdeña y la expansión mediterránea de la Corona de Aragón (1297-1314), 2 voll., Madrid, 1956; Eugenio DuPRE THESEIDER, Come Bonifacio VIII infeudò a Giacomo II il regno di Sardegna e Corsica, "VI Congresso Internazionale di Studi Sardi" (Cagliari. 1957), I, Cagliari, 1962, pp.89-101; Salvatore FODALE, Il regno di Sardegna e Corsica feudo della Chiesa di Roma (dalle origini al XIV secolo), in "Genova, Pisa e il Mediterraneo", cit., pp.515567. Sui rapporti tra la Corona d'Aragona e Genova la bibliografia è ampia; un inquadramento generale della problematica in Geo PISTARINO, Genova e Barcellona: incontro e scontro di due civiltà, "I Congresso storico Liguria-Catalogna" (Ventimiglia-Bordighera-Albenga-FinaleGenova, 1969), Bordighera, 1974, pp.81-122; IDEM, Sul tema dei rapporti tra Genovesi e Catalani, "Critica Storica”, IX (1972), pp.558-567; IDEM, Genova e la Corona d'Aragona (un "excursus" tra le fonti) in "Fonti e cronache italo-iberiche del Basso Medioevo. Prospettive di ricerca”. Firenze, 1984, pp. 95-117; Giuseppe MELoni, Genov'a e Aragona all'epoca di Pietro IV il Cerimonioso, 3 voll.. Padova, 1971-82; Blanca Garí, La comnotación estructural del
} 
Nei secoli XIV e XV la Sardegna catalano-aragonese divenne il punto di riferimento dei Corsi partigiani della Corona d'Aragona e il trait d'union tra questa e i suoi sostenitori in Corsica: la base più vicina per $\mathrm{i}$ contatti con gli alti funzionari regi catalano-aragonesi, un accogliente asilo politico per i fautori della Corona in lotta contro i Genovesi, una fonte di aiuti militari, mai sufficienti, per coloro che difendevano in patria le sorti del partito filoaragonese. Le pagine della cronaca rispecchiano questo ruolo assunto dall'isola in questo periodo.

Giovanni della Grossa mantiene un atteggiamento distaccato e neutrale nei confronti delle due potenze che si contendevano il dominio sulla Corsica: il suo desiderio sembra essere quello di vedere la sua isola pacificata sotto un governo forte, capace di mettere fine alle guerre e ai disordini, ma ritiene, con realistica visione dei fatti, che nè i Genovesi nè gli Aragonesi avessero un vero interesse al possesso dell'isola, se non per impedirvi l'insediamento della potenza rivale $^{33}$. In qualche punto della cronaca egli sembra propendere per la maggior fondatezza delle pretese aragonesi, sancite dall'infeudazione papale ${ }^{34}$, e spesso non tralascia di sottolineare, con tono di rassegnato distacco, come la Corona, nonostante le favorevoli circostanze verificatesi più volte, avesse sempre trascurato i suoi

conflicto entre Génova y la Corona de Aragón, in "Saggi e Documenti", 6, Genova, 1985, pp.283-306. Sulla Corsica nei secoli XIV-XV e il suo coinvolgimento nelle lotte tra la Corona d'Aragona e la repubblica ligure Giancarlo Sorgia, Corsica, Genova e Aragona nel Basso Medioevo, Sassari, 1965; Giovanna PETTI BALBI, Genova e Corsica, cit.

${ }^{33}$ Il cronista afferma che il re d'Aragona "non havea animo determinato di volere il dominio di Corsica se non quella poca dimostrazione e intratenimento dil suo titolo di Corsica, di quel modo che li signori di la famiglia di Cinarca suoi amici si la conquistassero e tenessero non lassandolo regnare al comune di Gienova..." Croniche, p.269, e che anche Genova "...solamente si contentava di tenere Corsica perchè altro principe non se ne appoderasse, massime quel d'Aragona...", p.263.

${ }^{34} \mathrm{Nel}$ delineare il mutamento della linea politica del nobile cinarchese Guglielmo della Rocca il cronista gli attribuisce un giudizio sulla legittimità dell'infeudazione pontificia della Corsica alla Corona d'Aragona, dal momento che l'isola non aveva un signore dai tempi di Arrigo Bel Messere e che i Genovesi l'avevano sempre trascurata, esprimendo, probabilmente, il suo stesso pensiero "E visto e considerato che il Papa avea dato Corsica e Sardegna al re di Aragona abenché il re, quando hebbe questa donazione, si pigliasse solamente Sardegna e di Corsica non fecie stima, li pareva a Guglielmo...che sarebbe stato infedeltà a farsi resistentia poiché la donazione dil Papa era justa..." e che i Genovesi "...non aveano mai cercata Corsica se da li Corsi non erano stati chiamati e pregati e non ne facevano conto niuno in Genova di Corsica”, Croniche, p. 200. 
diritti e anteposto altri problemi, ritenuti più importanti, alla conquista della Corsica $^{35}$.

Nella narrazione degli avvenimenti accaduti nel Trecento e negli anni in cui egli stesso visse, il cronista si sofferma sulle vicende delle principali famiglie signorili e sui loro rapporti con Genova e con la Corona d'Aragona, dilungandosi, in particolare, sulle imprese dei signori di Cinarca: Guglielmo della Rocca, suo figlio Arrigo della Rocca, Vincentello d'Istria. Egli accenna per la prima volta alla "nova pretensione" che sulla Corsica aveva la monarchia aragonese nelle pagine in cui racconta della conversione politica di Guglielmo della Rocca, nobile cinarchese che dopo aver governato l'isola per quattordici anni in nome dei Genovesi, si convertì alla causa aragonese dando impulso alla formazione del partito dei sostenitori della Corona d'Aragona nell'isola ${ }^{36}$. Al fine di chiarire i motivi che indussero il nobile corso al mutamento della sua linea politica, il cronista si sofferma su alcuni importanti avvenimenti accaduti in Sardegna a metà del XIV secolo, che facendo presagire una imminente conquista della Corsica da parte delle armate catalano-aragonesi, indussero il della Rocca a giurare fedeltà al re Pietro IV il Cerimonioso.

Giovanni della Grossa racconta che, mentre Guglielmo della Rocca governava in Corsica in nome di Genova, i Sardi si ribellarono alla Corona d'Aragona. La rivolta partì da Sassari, che era difesa da compagnie di soldati corsi, molti dei quali (ben settecento) vennero uccisi "tagliati a pezzi" dai rivoltosi, e si estese poi ad Alghero e a tutta l'isola. Questi fatti, secondo il della Grossa, avrebbero indotto il sovrano aragonese a passare in Sardegna con la sua armata e a porre l'assedio ad Alghero, "che si teneva per Genovesi”. Una grande battaglia navale tra i Catalani, sostenuti da alcune navi veneziane, e i Genovesi, avrebbe poi deciso le sorti della città che si arrese alla Corona d'Aragona, vincitrice sulla potenza rivale. In seguito a questi avvenimenti Guglielmo della Rocca si sarebbe recato nelle Bocche di

\footnotetext{
${ }^{35}$ Il cronista sottolinea spesso lo scarso interesse della Corona per l'isola, nonostante la leggittimità dei suoi diritti, e ricorda le numerose promesse di intervento mai mantenute, Croniche pp. 241, 262-263, 269, 272, 301, 310, 330, 364. La stessa accusa di disinteresse $\dot{\mathrm{e}}$ rivolta più volte anche a Genova, pp. 200, 263, 330.

${ }^{36}$ Su questo personaggio Jean André CANCELLIERI, voce Della Rocca, Guglielmo, in Dizionario Biografico, cit., pp. 310-313.
} 
Bonifacio, dove si trovava con la sua flotta il re aragonese, e gli avrebbe fatto atto di omaggio ${ }^{37}$.

Gli avvenimenti narrati corrispondono per grandi linee a quanto accadde in Sardegna tra il 1348 e il 1354: le rivolte di Sassari, la battaglia di Porto Conte e la conquista di Alghero da parte dei Catalano-Aragonesi, la ribellione della città e l'estensione della guerra a tutta l'isola sotto le insegne del giudicato d'Arborea, l'arrivo in Sardegna del re Pietro IV il Cerimonioso, il nuovo assedio e la definitiva conquista di Alghero ${ }^{38}$. Il cronista sintetizza rapidamente i fatti tacendo, per esempio, sulla ribellione di Alghero, che dopo la conquista catalana venne occupata dalle truppe arborensi del giudice Mariano IV, e li espone in un ordine cronologico impreciso, antecipando di un anno l'arrivo in Sardegna di Pietro IV il Cerimonioso, il quale, come sembra emergere dalla narrazione, avrebbe partecipato alla battaglia navale di Porto Conte, svoltasi in realtà nell'agosto del 1353, un anno prima dell'arrivo del sovrano nell'isola.

Il racconto della sconfitta dei Genovesi e della conquista di Alghero da parte dei Catalani assume nelle pagine della Cronaca un notevole rilievo, anche per l'influenza che questi avvenimenti ebbero, secondo il della Grossa, sulla formazione del partito filoaragonese corso. Il cronista concentra la sua attenzione sullo scontro tra le due maggiori potenze marittime del momento, Genova e la Corona d'Aragona, lasciando solo intravvedere la guerra, considerata niente di più di una rivolta, scoppiata proprio in quegli anni, tra la Corona ed i Sardi guidati dal giudice d'Arborea Mariano IV (che non viene mai menzoniato). Il ricordo di quegli avvenimenti e della grave sconfitta subita dalla repubblica ligure doveva essere ancora vivo ai tempi del della Grossa, che definisce la battaglia di Porto Conte una delle più crudeli di cui si fosse mai sentito parlare ${ }^{39}$.

\footnotetext{
${ }^{37}$ Croniche, pp. 199-201.

${ }^{38}$ Su questi avvenimenti Jeronimo ZURITA, Anales de la Corona de Aragón, ed. Canellas Lopez, 4, Zaragoza, 1978, I. VIII. cap. LII-LVII; Giuseppe MELONI, L'Italia medioevale nella cronaca di Pietro IV il Cerimonioso, Cagliari. 1980, pp. 87-117; IDEM, Genova e Aragona, cit.. I, pp. 149-214; Francesco Cesare Casula, La Sardegna aragonese, 2 voll., Sassari. 1990, I, pp.263-315. Sull'occupazione di Alghero da parte degli Arborea (ottobre 1353-novembre 1354) IDEM, Alghero arborense in "Alghero, la Catalogna", cit., pp.115-123. Da alcuni documenti dell'Archivio della Corona d'Aragona di Barcellona Guglielmo della Rocca risulta essere tra i signori corsi fedeli al re almeno dal 1345, Maria Giuseppina MELONI, Arrigo della Rocca: un nobile corso al servizio del re d'Aragona, "Mediovo. Saggi e Rassegne”, 18 (1993), pp.9-26.

${ }^{39}$ Croniche, p. 200
} 
Per quanto riguarda la rivolta di Sassari, non è chiaro a quale delle diverse ribellioni, o tentativi di ribellione, avvenuti negli anni 1348-1353, il cronista si riferisca ${ }^{40}$ : La notizia della presenza a Sassari di numerosi Corsi e del loro importante contributo alla difesa della città durante l'assedio dei Doria e la ribellione del 1348-49 è storicamente accertata, come si rileva dagli Anales de la Corona de Aragón di Jeronimo Zurita e da numerosi documenti dell'Archivio della Corona d'Aragona di Barcellona, ed è accertato altresì il ruolo da essi avuto durante il nuovo tentativo di rivolta organizzato dal corso Godiccello dell'Oliva nel $1353^{41}$. L'alto numero di Corsi che, secondo il racconto del della Grossa, sarebbero stati uccisi dai Sardi in rivolta testimonia, ancora una volta, la massiccia emigrazione che dalla Corsica si era diretta in quegli anni verso il nord della vicina isola.

A partire dalla seconda metà del XIV secolo assume grande rilievo nella narrazione del nostro cronista la figura del conte di Corsica Arrigo della Rocca: figlio di Guglielmo della Rocca egli, ancora più del padre, si legò alla causa aragonese, recandosi più volte in Catalogna a caldeggiare aiuti per la lotta contro i Genovesi, lotta che egli portò avanti per tutta la vita in nome della Corona, riusciendo in certi momenti, ad estendere il suo potere su quasi tutta l'isola ${ }^{42}$. Pur attraverso il tono distaccato della narrazione, Giovanni della Grossa rivela la sua ammirazione per questo personaggio: egli vede in Arrigo della Rocca una di quelle figure che avrebbero potuto prendere in mano il destino della Corsica se fossero state adeguatamente supportate "se avesse avuto forze e facoltà come infiniti principi del mondo, lui era homo da fare cose grandi e notabilissime in nel mondo" ma, nella sua lotta contro Genova, "lui non avea chi il

\footnotetext{
${ }^{40}$ Sulle rivolte di Sassari in questi anni Giuseppe Meloni, Genova e Aragona. cit.. I, pp. 21-54: Laura GalopPINI, Ricchezza e potere nella Sassari aragonese, Cagliari. 1989.

"Jeronimo ZURITA. Ancles. cit., 4. I.VIII. cap.XXVIII. Documenti dell Archivio della Corona d'Aragona che riportano questa notizia sono citati da Giuseppe MELONI. Genova $e$ Aragona cit.. I, p. 42, p. 48 e da Maria Giuseppina Meloni. Presenza corsa cit.. pp.9-33.

"Croniche. pp.216-237. Una biografia del personaggio è stata curata da Jean André CANCElLIERI, voce Della Rocca, Arrigo, in Dizionario Biografico, cit., pp. 299-302. Il titolo di conte di Corsica, come afferma Cancellieri e come appare dalla cronaca di Giovanni della Grossa, veniva conferito per acclamazione popolare, attraverso una particolare cerimonia, all autorità politica eminente nella Corsica medioevale, Croniche, p. 221. Sull'attività filoaragonese svolta in Corsica e in Sardegna dal della Rocca sulla base di documentazione dell'Archivio della Corona d'Aragona di Barcellona, Maria Giuseppina MELoni. Arrigo della Rocca, cit.
} 
soccorrisse...chè il re d'Aragona era molto lontano e in quelli tempi avea da fare per lui" ${ }^{43}$.

Dal racconto delle vicende di Arrigo della Rocca, che per quanto riguarda i suoi rapporti con la Corona d'Aragona trova riscontro quasi completo nelle fonti documentarie catalane, emerge in qualche modo anche il difficile momento attraversato in quegli anni (1370 circa-1401, anno della morte di Arrigo) dal regno di Sardegna. Il cronista sottolinea infatti, più volte, il contributo dato dal nobile corso alla guerra combattuta dai CatalanoAragonesi contro i Sardi, sostenuti da Genova". Mentre in Corsica "tenea la parte del re a bandere spiegate, anchora che il re non lo aiutava mai a le sue disgratie che li socciessero con li Gienovesi" Arrigo della Rocca "si corrispondea e servia al re di Aragona in quello tempo che lui potea e che il re li ordinava in Sardigna", facendogli "signalati servitii, difendendoli Sardegna da li Gienovesi". Questi servizi consistettero anche in un'intensa attività corsara svolta con alcune galere nel mare tra la Sardegna e la Corsica ${ }^{45}$. Il cronista mette in evidenza il ruolo avuto da Genova nel conflitto sardo-catalano: il suo interesse è rivolto soprattutto, come si è già notato, all'antagonismo tra la Corona d'Aragona e la repubblica ligure. Anche in Sardegna, dunque, Arrigo combatteva contro i Genovesi e i loro alleati sardi i quali, mettendo in serio pericolo il dominio aragonese in Sardegna, rischiavano di vanificare anche le aspirazioni del partito filoaragonese corso.

Ancora cenni alla guerra sardo-catalana si trovano nel racconto delle imprese di un altro personaggio che ebbe un ruolo importante nella storia corsa del ' 400 e che con lo stesso cronista ebbe stretti rapporti: Vincentello d'Istria $^{\text {th }}$.

Vincentello fu il principale protagonista della lotta antigenovese nella prima metà del XV secolo e colui che, con più tenacia, tenne alta in Corsica la bandiera aragonese, ottenendo dal re Alfonso il Magnanimo, nel 1418, il

\footnotetext{
${ }^{43}$ Croniche, p. 237.

${ }^{+4}$ Sulla guerra sardo-catalana Franceso Cesare Casula, La Sardegna aragonese, cit., I. pp 263-315; II, pp. 365 e ss.: Bruno ANATRA. La Sardegna dall'unificazione aragonese ai Savoia, Torino, 1987, pp. 50 e ss.

${ }^{45}$ Croniche, p. 230.

${ }^{46}$ Croniche, pp. 246-297.
} 
titolo di vicerè, che aggiunse a quello tradizionale di conte di Corsica ${ }^{47}$. Come si è detto all'inizio, Giovanni della Grossa fu a lungo al servizio di questo personaggio: visse dunque in prima persona molte delle vicende narrate, delle quali è senz'altro un testimone attendibile, o le apprese attraverso fonti dirette.

Secondo il racconto del cronista, Vincentello d'Istria, dopo aver rifiutato, al contrario di altri signori dell'Oltremonti, di sottomettersi a Genova, ed essersi rifugiato in Sardegna, a Longosardo, si guadagnò la stima del re d'Aragona Martino il Vecchio e di suo figlio Martino, re di Sicilia, attraverso l'attività corsara esercitata nei mari della Sicilia e della Sardegna contro i Genovesi e i Bonifacini, dapprima con galere armate a sue spese, poi a spese della Corona, insieme ad altri corsari catalani: "e la guerra allora era molto tra i Catalani e i Gienovesi, e loro andavano in corso contra a Gienovesi, e dopoi havere fatto molta presa, loro se ne andorno in Sardigna a invernare in Callari e in Laliera" 48 . Emerge qui, come già nel racconto delle imprese di Arrigo della Rocca, l'importanza assunta nel conflitto sardocatalano dalla guerra di corsa, attività condotta egregiamente dai nobili corsi filoaragonesi, che per alcune città del regno di Sardegna, come appunto Cagliari e Alghero, divenne in certe fasi della guerra particolarmente critiche per la Corona d'Aragona, l'unica fonte di approvvigionamento ${ }^{49}$.

Proseguendo nel racconto delle vicende di cui fu protagonista Vincentello d'Istria, il della Grossa ricorda la partecipazione del conte di Corsica al più importante fatto d'armi della guerra sardo-catalana nel XV secolo, la battaglia svoltasi a Sanluri nel giugno del 1409, che vide la sconfitta dei Sardi, schiacciati dalle armate aragonesi al comando del re

\footnotetext{
${ }^{47}$ Su questo personaggio A. AmBrosı. Un épisode de la guerre entre Gênes et Aragon au $X V$ siècle: Vincentello d'Istria. "Bulletin de la Societé des Sciênces Historiques et Naturelles de la Corse", XXXI (1911), pp.5-62. Sul titolo di vicerè di Corsica attribuitogli dal sovrano aragonese. Antonio Marongiu, La Corona d'Aragona e il regno di Corsica, "Archivio Storico di Corsica”, XI (1935), n. 4, pp. 481-501. Lattività svolta sotto le insegne della Corona d'Aragona da Vincentello d'Istria occupa largo spazio nelle cronache genovesi e in particolare. in Georgii et Johannis STELlaE, Annoles Gentenses, Rerum Italicarum Scriptores, vol. XVII. parte II, a cura di Giovanna Petti Balbi. Bologna. 1975.

${ }^{48}$ Croniche, pp.246-247.

${ }^{19}$ Sull importanza della guerra di corsa nei mari sardi durante la guerra sardo-catalana. Pinuccia F. Simbula, Corsari e pirati nei mari di Sardegna. Cagliari, 1990 (in particolare sull attivita corsara di Vincentello d'Istria pp.110-111). Anche il fratello di Vincentello, Giovanni d'Istria, fu un attivo corsaro al servizio della Corona d'Aragona nei mari sardi siciliani. Croniche, p. 252; p. 267.
} 
Martino di Sicilia ${ }^{50}$. Secondo il racconto del cronista, Vincentello, alla notizia dell'arrivo in Sardegna del re Martino, che già aveva servito come corsaro in Sicilia, raccolse quanti più uomini potè e si unì all'esercito regio che era inferiore di numero a quello dei "ribelli": durante lo scontro, secondo le notizie raccolte dal della Grossa, morirono settemila sardi, cifra che egli riporta però con il beneficio del dubbio perchè secondo altre voci ritenuta esagerata, e molti altri trovarono scampo nella fuga. Dopo la vittoria e la morte di Martino, che "amalò e morse in octo giorni di febre dil mese di agosto", Vincentello tornò in patria portando con sè sessanta balestrieri catalani che lo aiutarono a sottomettere alcuni signori riottosi alla sua autorită $^{51}$. Le scarne notizie riportate dal della Grossa sulla battaglia di Sanluri, come pure quella della morte di Martino il Giovane per febbri malariche nell'agosto del 1409 , corrispondono nella sostanza a quanto le fonti documentarie e narrative hanno tramandato su questo avvenimento, pur differendo in qualche particolare ${ }^{52}$; non è improbabile che le stesse notizie provenissero al cronista da fonti dirette, attraverso il racconto dello stesso Vincentello o di coloro che avevano preso parte alla battaglia.

L'ultimo cenno alle vicende politico-militari della Sardegna si ha in occasione del racconto del tentativo di conquista della Corsica attuato da Alfonso il Magnanimo nel 1420. Il della Grossa ricorda che in quell'anno, nel mese di settembre, prima di passare in Corsica, dove aveva preso Calvi e posto l'assedio a Bonifacio, il re Alfonso d'Aragona "con la sua armata di nave e galere" si era recato in Sardegna, aveva sottomesso Sassari e ridotto all'obbedienza tutta l'isola, che era stata definitivamente pacificata sotto la bandiera aragonese ${ }^{53}$.

\footnotetext{
${ }^{50}$ Su questo avvenimento Francesco Cesare Casula, La Sardegna aragonese, cit.. II. pp.: 521-556: IDEM, Ricerche archivistiche sulla battaglia di Sanluri. "Archivio Storico Sardo" XXXV (1986), pp. 123-133 in cui lo studioso sottolinea la mancanza di testimonianze dirette sullo svolgimento della battaglia dal momento che non sono giunte fino a noi le relazioni su questo fatto d'armi spedite da Martino il Giovane al padre dopo la vittoria. Tutti gli storici si rifanno quindi a Jeronimo ZURITA, Ancles, cit., 4. I. X, cap. LXXXVII, pp.912-916. Documenti sulla battaglia tratti dall'Archivio della Corona d'Aragona di Barcellona sono stati raccolti da Rafael CONDE y DELGADo DE Molina, La batalla de Sent Luri. Textos y Documentos, Sanluri, 1997.

${ }^{51}$ Croniche, pp.254-255.

${ }^{52}$ Secondo lo ZURITA. Anales, cit., 4. I. X, cap. LXXXVII, p.916, i Sardi morti a Sanluri furono cinquemila.

${ }^{53}$ Croniche, p.279. Sulla conclusione della guerra in Sardegna con la fine de facto e de jure del giudicato d.Arborea. Francesco Cesare Casula. La Sardegna aragonese, cit., II. pp. 157 e ss.; Bruno AnATRA, La Sardegna dall'unificazione. cit., pp. 157 e ss. Sul tentativo di
} 
Dopo la morte di Vincentello d'Istria, giustiziato nel 1434 dai Genovesi, la Sardegna, come appare anche dalle pagine della Cronaca, continuò ad essere il punto di riferimento per gli irriducibili sostenitori della Corona d'Aragona, sempre bisognosi di sostegno militare ed economico nella lotta contro Genova, rappresentata, a partire dal 1453, dal Banco di San Giorgio $^{54}$. Alfonso $\mathrm{V}$, sollecitato da numerose ambasciate provenienti dalla Corsica a non rinunciare al suo diritto sull'isola "che li suoi predecessori haveano acquistato et hauto justamente da la Chiesa romana, che era dapoi restato disprezzato" ${ }^{55}$, si limitò alla nomina di alcuni vicerè che condussero poco significative campagne militari contro i Genovesi. Dalla Sardegna arrivava, nel 1455, il nuovo vicerè aragonese di Corsica, Berenguer d'Erill, con una piccola flotta e "con dui ciento homini sardi, capo delli quali veneva il bisconte di Cagliari" per portare aiuto al capo del partito filoaragonese del momento, Raffaele da Leca, assediato dagli uomini dell'Officio di San Giorgio $^{56}$; e con la Sardegna quelle navi facevano la spola per rifornirsi di

conquista della Corsica da parte di Alfonso V il Magnanimo, Antonio Maronglu. La Corona d'Aragona cit.; IDEM. Il regno aragonese di Corsica e la convocazione parlamentare del 1420 in "Saggi di storia giuridica e politica sarda". Padova. 1975, pp.115-130. L'assedio di Bonifacio e la battaglia tra i Catalani e i Genovesi giunti a soccorrere la piazzaforte corsa sono descritti nelle cronache genovesi, Georgii et Johannis STEllaE. Annales Genuenses. cit, pp. 345-48; Agostino Giustiniani, Castigatissimi Annali con la loro copiosa tavola della eccelsa e illustrissima repubblica di Genova, Genoa, 1537 (rist. anast., Bologna, 1981), cc.CLXXXIv. CLXXXIV. Sulla politica mediterranea del sovrano aragonese e sul ruolo della Corsica in questa politica, soprattutto in funzione anti-genovese, Jaume VICENS VIVES, Els Trastamares. Segle $X V$, Barcelona, 1956; Ernesto PONTIERI, Alfonso V d'Aragona nel quadro della politica italiana del suo tempo, in "Estudios sobre Alfonso el Magnanimo", Barcelona, 1960, pp. 245-307; Eugenio DUPRÉ THESEIDER, La politica italiana di Alfonso il Magnanimo, "IV Congreso de Historia de la Corona de Aragón" (Palma de Mallorca, 1955). Ponencias, Barcelona. 1976. pp. 225-251; Coral Cuadrada Majó, Políica italiana de Alfonso V de Aragón (1420-1442), "Acta Historica et Archaeologica Mediaevalia”, VI (1986-87), pp. 7-8.

${ }^{54}$ Sui rapporti tra la Corona d'Aragona e i suoi sostenitori in Corsica in questo periodo Giancarlo SORGIA, Corsica, Genova, cit., pp. 59 e ss.; Luigi BULfERETTI, Le mire aragonesi sulla Corsica negli ultimi anni del regno di Alfonso il Magnanimo, "IV Congreso de Historia de la Corona de Aragón” (Palma de Mallorca, 1955), I, Palma de Mallorca, 1959, pp. 193-200. Sul governo del Banco di San Giorgio in Corsica Carlo BORNATE, La Corsica e il Banco di San Giorgio, "Archivio Storico di Corsica", V (1929), pp. 144-151; Flavia PERASSo, Genova e la Corsica nella seconda metà del Quatrocento, in "Genova, la Liguria e l'Oltremare tra Medioevo ed Età Moderna. Studi e ricerche d'archivio", I, Genova, 1974, pp. 41-120.

${ }^{55}$ Croniche, pp. 359-60.

${ }^{56}$ Giovanni della Grossa si dilunga sull'attività filoaragonese di Raffaele da Leca e sulle lotte da lui condotte in nome della Corona contro il Banco di San Giorgio, non tralasciando di sottolineare come egli fosse stato lasciato solo in questa lotta dagli Aragonesi dopo che questi. nel 1455, avevano stipulato una pace con Genova; lanno seguente, infatti, Raffaele da Leca venne catturato dai Genovesi i quali. secondo il raccondo del cronista, lo squartarono. Io salarono come un porco e mandarono un quarto del suo corpo a Calvi. uno a Bonifacio. uno a 
vettovaglie e armi ${ }^{57}$. Come attestano i documenti, Alfonso il Magnanimo ordinò di fornire gli aiuti necessari a quest'impresa ai più alti funzionari regi del regno di Sardegna, tra i quali il governatore del Capo di Cagliari e Gallura Jaume Carroç, conte di Quirra, il "visconte di Cagliari" citato dal della Grossa ${ }^{58}$.

La Sardegna divenne, in quegli anni (1457-1464) la base di appoggio di numerosi fuoriusciti corsi, molti dei quali appartenenti alla famiglia dei Cinarchesi, che furono oggetto di una vera e propria persecuzione da parte dei Genovesi, decisi a sottomettere definitivamente la riottosa feudalità corsa. Il della Grossa scrive che sotto la protezione del vicerè di Sardegna si formò nell'isola (nel nord dell'isola, probabilmente a Sassari) una piccola comunità di esuli corsi capeggiata da uno dei più tenaci partigiani della Corona, Paolo della Rocca, che raccolse presso di se parenti ed amici sfuggiti alle persecuzioni genovesi in attesa di tempi migliori per fare ritorno in patria ${ }^{59}$

La morte di Alfonso il Magnanimo segnò anche la fine delle pretese aragonesi sulla Corsica ma non, ancora per qualche tempo, delle speranze dei sostenitori della Corona nell'isola, impegnati in una strenua lotta contro l'Officio di San Giorgio. L'ultima ambasciata, ricordata dal nostro cronista, per richidere aiuto al sovrano aragonese fu quella compiuta a Valenza, presso il re Giovanni II, dal signore cinarchese Giocante da Leca il quale però "non ebbe ajuto dal re se non che, solamente per mostrarli qualche sua voluntà, il fecie suo cavallero" e gli promise la concessione di alcune rendite in Sardegna. E' il consueto atteggiamento, più volte denunciato dal della Grossa, dei sovrani aragonesi, che cercarono di mantenere l'appoggio dei signori cinarchesi lusingandoli con titoli e promesse in modo da indurli a continuare la lotta contro Genova, per impedire alla principale rivale della Corona nel Mediterraneo il pacifico possesso dell'isola. E, ancora una volta, la Sardegna divenne il rifugio dei partigiani della Corona abbandonati a se

Corte e uno a Biguglia mentre la testa venne posta sul castello di Cinarca, Croniche, pp. 370 399.

${ }^{57}$ Croniche, pp. 389-91.

${ }^{58}$ Giancarlo Sorgia, Corsica, Genova, cit., p .76.

${ }^{51}$ Croniche, pp. 403, 414, 417, 424. Oltre ai membri della casata dei Cinarchesi, la Sardegna probabilmente accolse, in quegli anni, anche molta gente comune che cercò di sfuggire alla guerra e alla miseria emigrando. Dice infatti il della Grossa che, in quel periodo, più di un terzo della popolazione dello stato cinarchese lasciò la Corsica. pp. 393-94. 
stessi: anche Giocante da Leca, infatti, dice il cronista, deluso dall'atteggiamento del re, se ne andò a Sassari con tutta la sua famiglia ${ }^{(t)}$.

Con il racconto delle lotte tra i Cinarchesi, rientrati dalla Sardegna approfittando del malcontento della maggior parte della popolazione, contro l'Officio di San Giorgio, e dei contrasti tra quest'ultimo e Tommasino Fregoso, nominato governatore di Corsica nel 1464 da un'assemblea di signori in contrapposizione all'Officio stesso, si chiude la cronaca di Giovanni della Grossa ${ }^{61}$.

Si può affermare, in conclusione, che seppure l'opera del cronista corso presenti i limiti che si sono evidenziati riguardo alla sua utilizzazione come fonte storica almeno fino al XIII secolo, essa riveste ugualmente un certo interesse per quanto riguarda il periodo giudicale sardo perché rivela, anche attraverso il filtro di leggende e tradizioni, la conoscenza e l'immagine che si aveva in Corsica, nel XV secolo, di questo fondamentale momento della storia sarda. L'opera del della Grossa è invece una fonte importante, a partire dal XIV secolo, riguardo ai rapporti tra la feudalità corsa e la Corona d'Aragona e al ruolo di tramite esercitato, in questi rapporti, dal regno di Sardegna catalano-aragonese, le cui vicende sono seguite con attenzione dal cronista nella più ampia ottica della lotta tra la Corona d'Aragona e Genova per il dominio sul Mediterraneo.

\section{RÉSUMÉ}

La chronique de Giovanni della Grossa, écrite dans la seconde moitié du XV siècle. est la plus ancienne chronique de la Corse médiévale. Dans cette ouvrage, que raconte Thistoire de la Corse de ses origines a l'année 1464, il y a plusieurs références à la voisine Sardaigne: à travers le récit de l'histoire de la Corse on peut extrapoler des episodes d'histoire sarde. Ce travail examine les raisons et les façons de la presence de la Sardaigne dans le récit du chroniqueur corse. Mème si on ne peut pas l'utiliser comme source historique pour les siécles du IX jusqu'au XIII, cette chronique nous montre l'image que on avait en Corse de la Sardaigne "giudicale"; au contraire, le récit de Giovanni della Grossa est une

\footnotetext{
${ }^{(3)}$ Cromiche. pp. 420-21.

"Le vicende della Corsica dal 1464 al 1481 sono state studiate, sulla base della ricca documentazione conservata nell Archivio di Stato di Milano, da Riccardo Musso, Il dominio sforzesco in Corsica (1464-1481). "Nuova Rivista Storica”. LXXVIII (1994), fasc.III, pp.531588; LXXIX (1995), fasc.I, pp. 27-76.
} 
source importante pour la question des relations entre la Corse, la Couronne d'Aragon et la Sardaigne catalan-aragonese pandant le XIV et le XV siècle.

\section{SUMMARY}

The chronicle written by Giovanni della Grossa in the middle of the fifteenth century. is the oldest chronicle about medieval Corsica. In his work, that relates the history of Corsica from its origins to 1464 . the writer refers to the nearby island of Sardinia many times. So, through the narration of Corsica's history, some episodes emerge of Sardinian history. The article examines the motives and the ways of the Sardinia's presence in the corse chronicle. Even if this chronicle can't be used as a historical source for the IX to the XIII centuries, there emerges from it a picture of the "giudicale" Sardinia, while the story of the writer della Grossa is an important source for the relations between Corsica, the Crown of Aragon and the catalan-aragonese Sardinia in the XIV-XV centuries. 